\title{
Emotion Recognition Index através da Teoria de Resposta ao Item
}

\section{Emotion Recognition Index item analysis: validation study from Item Response Theory perspective}

\author{
Ricardo Alberti', Fernando de Jesus Moreira Junior", \\ Silvio José Lemos Vasconcellos'", Felipe Coelho Argolo'v, Nathalia Ruviarov, \\ Marcielle Toaldo da Silvavi
}

\begin{abstract}
RESUMO
Introdução: O Emotion Recogntion Index (ERI) foi desenvolvido para avaliar a percepção emocional. Seus itens foram selecionados usando Teoria Clássica dos Testes. Objetivo e Métodos: Avaliar o desempenho dos itens do ERI sob perspectivas psicométricas atuais, usando Teoria da Resposta ao Item (TRI) por meio de um modelo logístico de dois parâmetros. Resultados: Foram retirados 12 itens do Módulo Face para que a premissa de unidimensionalidade fosse cumprida. Foram eliminados 20 itens do Módulo Voz por apresentarem baixos valores no parâmetro discriminação. Ambos os módulos apresentarem curvas de informação com pico em valores baixos para o traço latente. Discussão: Os achados sugerem que o ERI possui, predominantemente, itens de baixa dificuldade, sendo uma ferramenta melhor empregada para avaliar indivíduos com habilidade baixa em reconhecimento de expressões faciais. Para desenvolver ferramentas mais balanceadas em futuros estudos, os autores sugerem o uso de TRI para os instrumentos voltados a medir percepção emocional.
\end{abstract}

Palavras-chave: Teoria de resposta ao Item; Emotion Recognition Index; Emoções; Expressões faciais; Comportamento não-verbal.

\begin{abstract}
Background: The Emotion Recognition Index (ERI) is a psychometric tool develop to evaluate emotional perception. Its items were selected using Classical Tests Theory. Objectives and Methods: Evaluate ERI items performance under current psychometric theories, using Item Response Theory (IRT) thought a two parameter logistic model. Results: 12 items were removed from the Face Module to fulfill the unidimensionality assumption. 20 items were eliminated from the Voice Module because they presented low discrimination values. Both modules presented information curves peaks among low latent trait values. Discussion: Findings suggest ERI is mostly composed of low difficulty items. ERI is best used to evaluate individuals with low emotional perception. Authors suggest further studies should use IRT to select items for instruments evaluating emotional perception.
\end{abstract}

Keywords: Item Response Theory; Emotion Recognition Index; Emotions ;Facial expressions; Nonverbal behavior.

\footnotetext{
' Universidade Federal de Santa Maria, Santa Maria, Brasil. E-mail:r-alberti@live.com

"Universidade Federal de Santa Maria, Santa Maria, Brasil. E-mail:fmjunior777@yahoo.com.br

III Universidade Federal de Santa Maria, Santa Maria, Brasil. E-mail:silviojlvasco@hotmail.com

Iv Universidade Federal de São Paulo, São Paulo, Brasil. E-mail:felipe.c.argolo@prontonmail.com

v Universidade Luterana do Brasil, Brasil. E-mail:r-alberti@live.com

v' Universidade Federal de Santa Maria, Santa Maria, Brasil. E-mail: r-alberti@live.com
} 


\section{INTRODUÇÃO}

Conceitualmente emoção é considerada como um elemento do conjunto genérico de estados afetivos e um processo que envolve múltiplas variáveis. Conforme Scherer (2007), normalmente a emoção é breve e ocorre em razão de um estímulo interno ou externo. Quando as mesmas manifestações corporais aparecem em diferentes culturas elas passam a ser chamadas de emoções básicas sendo exibidas da mesma forma, desde crianças até idosos (EKMAN, 2003).

Emoções básicas são respostas fisiológicas involuntárias que são universalmente compartilhadas por nossa espécie - visualmente distinguível e pré-estabelecidas por estímulos que influenciaram nossa evolução e podem também ser moldadas por experiências de vida. O reconhecimento da expressão de emoções é um dos componentes básicos para a comunicação não-verbal (KNAPP; HALL, 2002). Problemas com o processamento de emoções faciais podem, assim, levar a más interpretações de determinadas situações sociais ou comportamentos inapropriados. Vários estudos recentes sugerem que pessoas com transtornos psiquiátricos, como depressão, ansiedade, bipolaridade, psicopatia e outros, apresentam deficiências no reconhecimento de emoções faciais (KANG et. al., 2019).

Existem hoje estados emocionais que são entendidos pelas teorias psicoevolucionistas como reflexo da evolução das espécies, ou seja, como respostas adaptativas a situações que ocorrem no meio. Cada estado emocional possuía uma funcionalidade adaptativa e expressão típica e as emoções humanas teriam evoluído de um conjunto finito desses estados (EKMAN, 2003; GAZZA-NIGA; IVRY; MANGUN, 2006; LUNDQVIST; ÖHMAN, 2005; PLUTCHIK, 2002). No que diz respeito à expressão de emoções, vários aspectos do comportamento expresso podem ser levados em conta.

As expressões emocionais devem ser entendidas nas suas funções conforme ressalta Darwin e, portanto, são destacadas pelo seu valor de sobrevivência. Desde então entende-se que, embora certas formas de manifestação das emoções possam ser aprendidas, existem expressões, especialmente as faciais, que são inatas (MIGUEL, 2015). Nesse contexto, uma expressão emocional corresponde àquilo que é demonstrado a outras pessoas, voluntária ou involuntariamente (PICARD, 1997). 
De acordo com exposto, pode-se salientar que o debate sobre a definição, a conceitualização e os aspectos físicos, psíquicos e comportamentais que estão envolvidos na emoção surge inicialmente com a ideia de tripartição cognição-emoçãomotivação de Platão, incluindo divisão mente-corpo de Descartes, a universalidade e o evolucionismo de Darwin e a importância da percepção dos estados corporais de William James. Os modelos contemporâneos de estudo das emoções, que englobam conceituações epistemológicas, reações comportamentais e fisiológicas, juntamente com as variáveis correlatos cerebrais (SANTOS, 2017).

Em encontro a nossas vivencias, no cotidiano, percebemos variados sinais nãoverbais, no que diz respeito as emoções e sua utilização como parte relevante a transmissão de mensagens entre os indivíduos, como também uma maneira de comunicação, servindo como questões complementares às trocas de mensagens verbais transmitidas. Podemos perceber, que as expressões faciais, gestos, dicção, postura corporal e percepções são exemplos nítidos de comunicação não-verbal, utilizados diariamente por as mais variadas culturas e por diferentes espécies distintas (VIEIRA, 2015).

Cada emoção também gera um padrão único de sensações em nosso corpo. Ao nos familiarizamos com elas, podemos ficar cientes, desde o início, de nossa resposta emocional, a fim de termos alguma chance de escolher se conservamos a emoção ou se interferimos nela, como, também representa sinais únicos, na fisionomia e na voz (EKMAN, 2011). Por esta razão as emoções faciais possuem um papel de extrema importância nas relações interpessoais. É através dela que acabamos por perceber, reconhecer, interpretar, e produzir, as respostas e mensagens emocionais, sendo este o motivo da grande, importância desta, nas diferentes interações sociais. Ressaltando que é através da observação da expressão não-verbal alheia, que podem-se deduzir informações sobre seu estado emocional, suas intenções e, inclusive, suas reações (VIEIRA, 2015).

Apesar do extenso e crescente volume de publicações relacionadas a essa temática segundo Porter et. al., (2012), existem poucas ferramentas validadas para testar as habilidades individuais no reconhecimento de emoções. Além disso, revela-se 
predominante o uso da Teoria Clássica dos Testes (TCT) para a validação de instrumentos empregados para esses fins (BIEHL et al. 1997, SCHERER, SCHERER, 2011).

O Emotion Recognition Index (ERI) é instrumento em forma de software desenvolvido e validado na pelo Swiss Center for Affective Sciences da Universidade de Geneva (SCHERER, 2007), composto de duas subescalas, uma para o reconhecimento de emoções através de expressões faciais (FACIAL-I) e outra para o reconhecimento por intermédio de estímulos verbais (VOCAL-I). Cada uma contém 30 itens.

A Teoria da Resposta ao Item (TRI) é um método que estabelece a correspondência entre variáveis latentes e suas manifestações (DE AYALA, 2009), ou seja, uma forma de representar a relação entre a probabilidade de um indivíduo dar uma resposta a um item e o seu traço latente ou habilidade (ANDRADE; TAVARES; VALLE, 2000). São exemplos de traços latentes: proficiência, habilidade, grau de satisfação, importância atribuída, nível de ansiedade, grau de usabilidade, etc. (MOREIRA JUNIOR, 2014).

Como descrito no estudo de validação original (SCHERER, SCHERER, 2011), o ERI foi desenvolvido para avaliar a competência em percepção emocional (emotional perception competence), descrito como um dos domínios da competência emocional (emotional competence), um conceito proposto previamente (SCHERER, 2007).

O objetivo desse trabalho é avaliar o ERI sob a perspectiva da Teoria da Resposta ao Item (TRI) por meio de um Modelo Logístico Unidimensional de Dois Parâmetros (ML2), uma vez que competência emocional se adequa às características de um traço latente. O ML2 avalia a discriminação (a) e a dificuldades (b) dos itens. Foram analisados os itens que compõem o ERI e observadas as consequências da seleção dos itens de melhor performance para compor o instrumento.

\section{MÉTODO}

O recrutamento dos participantes ocorreu em dois centros, na Universidade Federal de Santa Maria (FSM), Santa Maria, Rio Grande do Sul, Brasil, e na Universidade Federal da Bahia (UFBa), Salvador, Bahia, Brasil. Candidatos foram recrutados de maneira voluntária, após preenchimento de termo de consentimento informado. Os 
meios de divulgação foram listas de e-mail, cartazes dispostos nos campi e convites em sala de aula.

As imagens foram apresentadas por meio de um retroprojetor de multimídia (LCD, resolução SVGA $800 \times 600$ ) para grupos de até 20 pessoas e os sons foram apresentados em amplificadores de $20 \mathrm{~W}^{1}$. Em todas as aplicações, realizou-se uma testagem das condições de visibilidade para todos os participantes. Foi realizada uma coleta preliminar com um grupo de 13 pessoas, para detecção de possíveis problemas na aplicação dos instrumentos e no preenchimento dos questionários pelos indivíduos. Os resultados obtidos por este grupo foram bastante próximos aos descritos pelo estudo de validação original (Média de acertos de $71 \%$ vs. $73 \%$ do estudo original em Scherer e Scherer, 2001) e não foram reportadas dificuldades para interpretação e visualização dos itens. Os resultados da coleta preliminar foram descartados da análise final.

Para verificar a unidimensionalidade dos dados, foi feita análise fatorial baseada na matriz das correlações tetracóricas. Itens foram eliminados progressivamente segundo os resultados mais baixos do Coeficiente de Correlação Ponto-Bisserial (CCPC) até que fosse obtida um dimensão predominante, suposição exigida para o ajuste de modelos unidimensionais da TRI. A estimativa foi feita considerando percentil 95 do percentual randômico da variância por item (TIMMERMAN; LORENZA-SEVA, 2011).

Na modelagem do ML2, foi usado o método da Máxima Verossimilhança para estimativa dos parâmetros. O traço latente foi estimado pelo método bayesiano da Esperança à Posteriori (EAP). Após a modelagem, itens com baixa discriminação foram progressivamente eliminados até que todos os itens tivessem discriminação maior que 0,64, ponto de corte para boa discriminação, segundo critério de Baker (1992). Itens restantes tiveram seus parâmetros estimados novamente.

Os itens removidos durante a análise foram analisados qualitativamente por um especialista certificado em Facial Action Coding System (EKMAN; FRIESEN, 1978) para identificar possíveis fatores relacionados aos itens com parâmetros que tiveram estimativas inadequadas. Foram usados os pacotes Itm (RIZOPOULOS, 2013), irtoys

${ }^{1}$ Condições operacionais impossibilitaram a aplicação do módulo Voz em um dos centros, resultando em perda de 52 indivíduos. 
(PARTCHEV, 2015) do Ambiente e Linguagem de Programação $R$ (R DEVELOPMENT CORE TEAM, 2012) e o software FACTOR, versão 9.2 (LORENZO-SEVA; FERRANDO, 2013).

\section{RESULTADOS}

Um total de 247 indivíduos foi recrutado. Destes, 195 estudantes da UFSM, 55 estudantes e profissionais vinculados à UFBa. Características gerais da amostra estão dispostas na Tabela 1.

Tabela 1 - Sexo, idade e composição por ocupação da amostra analisada

\begin{tabular}{lc}
\hline Característica & Dados $(\mathrm{n}=247)$ \\
\hline Sexo & $152(61,5)$ \\
Homens & $93(38,5)$ \\
Mulheres & \\
Idade & $22,34 \pm 5,03$ \\
Média \pm Desvio Padrão & \\
Grupo & $195(78,9)$ \\
Centro UFSM & $52(20,1)$ \\
Centro UFBa & Todos os dados são apresentados como n (\%), exceto se especificado.
\end{tabular}

\subsection{Módulo Face}

A Tabela 2 apresenta o percentual de acertos e erros dos 30 itens utilizados na avaliação. Pode-se verificar que há mais itens fáceis do que difíceis, segundo o percentual de acerto/erro. Nota-se, por exemplo, que 9 itens (30\%) tiveram um percentual maior que $90 \%$ de acertos. O item Face6 é o que possui maior percentual de acertos (99,5\%), por outro lado, o item Face2 é o que possui maior percentual de erros $(96,4 \%)$. 
Tabela 2 - Percentual de acertos e erros dos itens

\begin{tabular}{lcc}
\hline Item & Acertos (\%) & Erro $(\%)$ \\
\hline Face1 & 97,4 & 2,6 \\
Face2 & 3,6 & 96,4 \\
Face3 & 95,4 & 4,6 \\
Face4 & 91,3 & 8,7 \\
Face5 & 33,9 & 66,2 \\
Face6 & 99,5 & 0,5 \\
Face7 & 59,5 & 40,5 \\
Face8 & 38,0 & 62,1 \\
Face9 & 40,5 & 59,5 \\
Face10 & 96,4 & 3,6 \\
Face11 & 75,9 & 24,1 \\
Face12 & 18,5 & 81,5 \\
Face13 & 82,1 & 18,0 \\
Face14 & 92,8 & 7,2 \\
Face15 & 87,2 & 12,8 \\
Face16 & 93,9 & 6,2 \\
Face17 & 84,1 & 15,9 \\
Face18 & 92,8 & 7,2 \\
Face19 & 89,2 & 10,8 \\
Face20 & 32,8 & 67,2 \\
Face21 & 77,4 & 22,6 \\
Face22 & 84,6 & 15,4 \\
Face23 & 23,6 & 76,4 \\
Face24 & 59,5 & 40,5 \\
Face25 & 79,0 & 21,0 \\
Face26 & 67,2 & 32,8 \\
Face27 & 32,8 & 67,2 \\
Face28 & 17,4 & 82,6 \\
Face29 & 89,2 & 10,8 \\
Face30 & 96,4 & 3,6 \\
\hline & &
\end{tabular}

$\mathrm{Na}$ análise fatorial, observou-se que o primeiro fator explicava apenas 12,5\% da variância dos dados, com a existência de 6 a 8 dimensões, conforme análise fatorial exposta na Tabela 3. Após a exclusão de 12 itens, devido à baixos valores do CPCC, foi possível obter uma variância explicada de $21,5 \%$ para o primeiro fator, sendo esse caracterizado como fator dominante.

Após a verificação da Unidimensionalidade, os 18 itens resultantes foram utilizados na análise da TRI. Todos itens apresentaram bons resultados exceto o item Face11, que apresentou um valor um pouco baixo de discriminação $(0,50)$, segundo Baker (1992), sendo, assim, eliminado. Os 17 itens restantes foram modelados pela TRI e o valor estimado dos parâmetros de Discriminação e de Dificuldade, e seus respectivos Erros Padrões (EP) são apresentados na Tabela 3. As Curvas Características 
dos Itens (CCI's) são apresentadas na Figura 1(a), enquanto que as Funções de Informação dos Itens (FII's) são apresentadas na Figura 1(b).

Observa-se que todos os 17 itens estão adequadamente ajustados. O item Face16 foi o item com maior discriminação $(2,60)$, enquanto que o item Face7 foi o item com a menor discriminação $(0,78)$. O item Face24 foi o item com maior dificuldade ($0,49)$, enquanto que o item Face4 foi o item com a menor dificuldade de acerto $(-3,19)$.

Tabela 3 - Parâmetros de Discriminação (a) e de Dificuldade (b) dos itens e seus Erros Padrões (EP) para Módulo Voz.

\begin{tabular}{lcccc}
\hline \multirow{2}{*}{ Item } & \multicolumn{2}{c}{ Discriminação (a) } & \multicolumn{2}{c}{ Dificuldade (b) } \\
\cline { 2 - 5 } & Valor & Erro Padrão & Valor & Erro Padrão \\
\hline Face1 & 1,79 & 0,65 & $-2,84$ & 0,60 \\
Face4 & 0,82 & 0,32 & $-3,19$ & 1,07 \\
Face7 & 0,78 & 0,24 & $-0,55$ & 0,25 \\
Face10 & 2,01 & 0,64 & $-2,47$ & 0,43 \\
Face13 & 0,95 & 0,28 & $-1,87$ & 0,48 \\
Face14 & 2,52 & 0,72 & $-1,80$ & 0,26 \\
Face15 & 1,06 & 0,32 & $-2,15$ & 0,51 \\
Face16 & 2,60 & 0,73 & $-1,89$ & 0,26 \\
Face17 & 2,32 & 0,65 & $-1,23$ & 0,20 \\
Face18 & 1,64 & 0,46 & $-2,16$ & 0,39 \\
Face21 & 0,94 & 0,27 & $-1,53$ & 0,39 \\
Face22 & 1,09 & 0,31 & $-1,89$ & 0,44 \\
Face24 & 0,89 & 0,26 & $-0,49$ & 0,22 \\
Face25 & 1,15 & 0,31 & $-1,42$ & 0,32 \\
Face26 & 0,84 & 0,25 & $-0,98$ & 0,31 \\
Face29 & 0,84 & 0,31 & $-2,83$ & 0,88 \\
Face30 & 1,38 & 0,50 & $-3,00$ & 0,74 \\
\hline
\end{tabular}

Figura 1 - Curvas Características dos Itens - CCl's (a) e Função de Informação dos Itens - FII's (b)

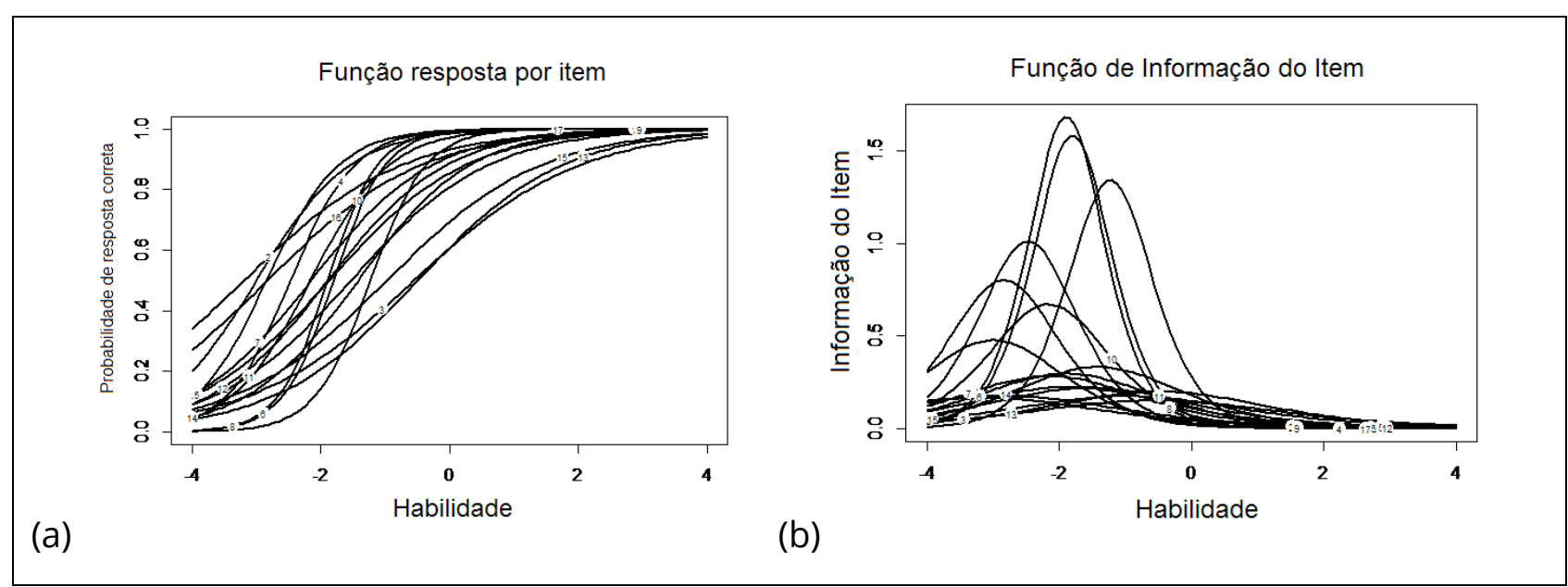


A Função de Informação Total (FIT), que é o acumulado das FII, está apresentada na Figura 2, observa-se que a maior parte da informação concentra-se entre -4 e 0 , sugerindo que o ERI é mais adequado para medir indivíduos que têm baixa habilidade em identificar as emoções faciais. Isso é consequência direta da inexistência do número de itens com parâmetros de dificuldade positivos.

Figura 2 - Função de Informação do Teste

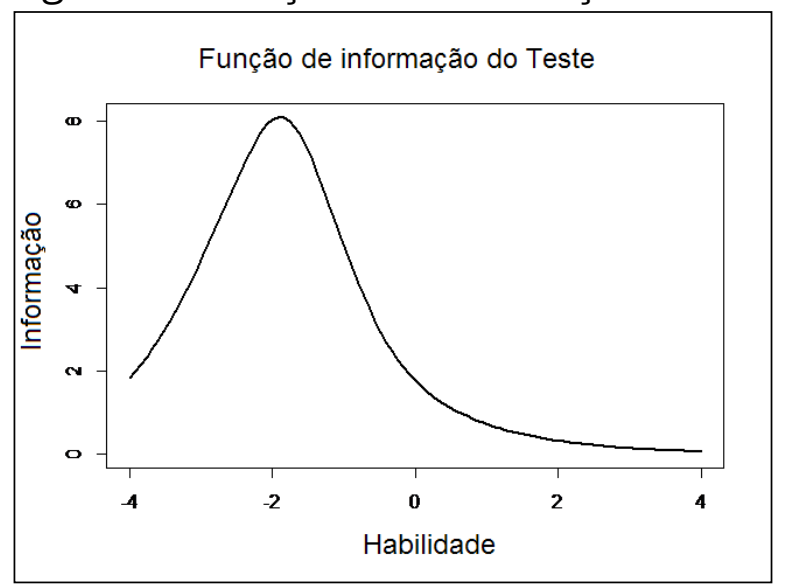

A Figura 3 apresenta o histograma (a) e o erro padrão (b) do traço latente. Observa-se que enquanto a maior parte da informação se concentra à esquerda, abaixo da média da escala (Figura 2), a maior parte dos indivíduos se concentra no meio, em torno da média da escala (Figura 4(a)).

É possível que a existência de indivíduos com traço latente acima do valor 1,5, na amostra não tenha sido estimada pela carência de itens capazes discriminar altos valores. Quanto ao erro padrão (SEM), eixo y do gráfico da Figura 4(b), observa-se que esse aumenta à medida que a informação (Figura 2).

Figura 3 - (a) Histograma e (b) Erro Padrão do traço latente

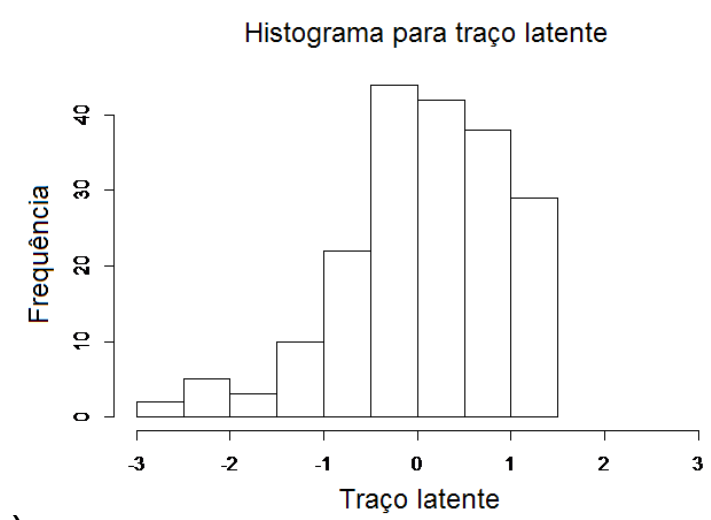

(a)

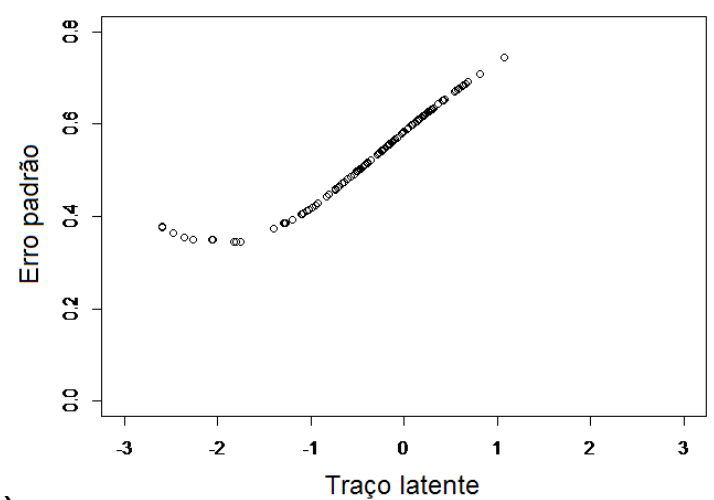

(b) 
3.2 Módulo Voz.

A Tabela 4 apresenta o percentual de acertos e erros dos 30 itens utilizados na avaliação. Observando-se a Tabela 3, pode-se verificar que há uma quantidade equilibrada de itens fáceis, médios e difíceis, segundo o percentual de acerto/erro. 0 item Voz8 é o que possui maior percentual de acertos (93,3\%), por outro lado, o item Voz25 é o que possui maior percentual de erros $(95,4 \%)$.

Tabela 4 - Percentual de acertos e erros dos itens

\begin{tabular}{ccc}
\hline Item & \% de acerto & \% de erro \\
\hline Voz1 & 33,9 & 66,2 \\
Voz2 & 55,9 & 44,1 \\
Voz3 & 73,9 & 26,2 \\
Voz4 & 39,0 & 61,0 \\
Voz5 & 69,7 & 30,3 \\
Voz6 & 57,4 & 42,6 \\
Voz7 & 45,6 & 54,4 \\
Voz8 & 93,3 & 6,7 \\
Voz9 & 67,2 & 32,8 \\
Voz10 & 46,7 & 53,3 \\
Voz11 & 64,1 & 35,9 \\
Voz12 & 34,9 & 65,1 \\
Voz13 & 76,4 & 23,6 \\
Voz14 & 46,7 & 53,3 \\
Voz15 & 74,4 & 25,6 \\
Voz16 & 5,6 & 94,4 \\
Voz17 & 47,7 & 52,3 \\
Voz18 & 70,3 & 29,7 \\
Voz19 & 65,1 & 34,9 \\
Voz20 & 68,2 & 31,8 \\
Voz21 & 84,6 & 15,4 \\
Voz22 & 5,6 & 94,4 \\
Voz23 & 52,8 & 47,2 \\
Voz24 & 28,7 & 71,3 \\
Voz25 & 4,6 & 95,4 \\
Voz26 & 19,5 & 80,5 \\
Voz27 & 83,1 & 16,9 \\
Voz28 & 61,0 & 39,0 \\
Voz29 & 85,6 & 14,4 \\
Voz30 & 33,3 & 66,7 \\
\hline & &
\end{tabular}

O resultado encontrado mostrou que o primeiro fator explica apenas $9,4 \%$ da variância dos dados. Apesar do baixo percentual de explicação do primeiro fator, considerando o percentil 95 como critério, os resultados indicam que o construto pode ser representado por um fator dominante. 
Após a verificação da Unidimensionalidade, os 30 itens foram utilizados na análise da TRI. Os resultados para a primeira análise estão dispostos na Tabela 5. Os resultados mostram predominância de valores baixos para discriminação e erros padrões altos para o parâmetro de Dificuldade. Considerando o critério de Baker (1992), que considera aceitável itens com discriminação maior que 0,64, foi realizada a remoção sequencial de itens com discriminação baixa, obtendo-se um total de 20 itens eliminados. Os resultados para as novas estimativas dos parâmetros são apresentados na Tabela 6. As CCl's dos itens são apresentadas na Figura 4(a), enquanto que as Fll's são apresentadas na Figura 4(b).

Tabela 5 - Parâmetros iniciais de Discriminação (a) e de Dificuldade (b) e seus Erros Padrões (EP) do Módulo Voz.

\begin{tabular}{lllll}
\hline \multirow{2}{*}{ Item } & \multicolumn{3}{l}{ Discriminação } & \multicolumn{2}{l}{ Dificuldade } \\
\cline { 2 - 5 } & $\mathrm{a}$ & $\mathrm{EP}(\mathrm{a})$ & $\mathrm{b}$ & $\mathrm{EP}(\mathrm{b})$ \\
\hline Voz1 & 0,33 & 0,22 & 2,08 & 1,37 \\
Voz2 & 0,09 & 0,19 & $-2,63$ & 5,75 \\
Voz3 & 0,15 & 0,21 & $-6,99$ & 9,98 \\
Voz4 & 0,51 & 0,23 & 0,94 & 0,48 \\
Voz5 & 0,28 & 0,21 & $-2,99$ & 2,23 \\
Voz6 & 0,71 & 0,24 & $-0,46$ & 0,26 \\
Voz7 & 0,30 & 0,20 & 0,60 & 0,62 \\
Voz8 & 0,90 & 0,39 & $-3,31$ & 1,16 \\
Voz9 & 0,60 & 0,23 & $-1,28$ & 0,51 \\
Voz10 & 0,04 & 0,19 & 3,03 & 13,52 \\
Voz11 & 0,93 & 0,27 & $-0,73$ & 0,25 \\
Voz12 & $-0,15$ & 0,20 & $-4,19$ & 5,61 \\
Voz13 & $-0,21$ & 0,24 & 5,68 & 6,46 \\
Voz14 & 0,33 & 0,20 & 0,41 & 0,50 \\
Voz15 & 0,60 & 0,24 & $-1,92$ & 0,72 \\
Voz16 & $-0,42$ & 0,39 & $-6,87$ & 6,08 \\
Voz17 & 0,77 & 0,25 & 0,14 & 0,21 \\
Voz18 & 0,75 & 0,25 & $-1,28$ & 0,42 \\
Voz19 & 0,69 & 0,24 & $-1,00$ & 0,38 \\
Voz20 & 0,19 & 0,20 & $-4,00$ & 4,23 \\
Voz21 & 1,73 & 0,49 & $-1,44$ & 0,26 \\
Voz22 & $-0,22$ & 0,39 & $-13,12$ & 23,42 \\
Voz23 & 0,43 & 0,21 & $-0,27$ & 0,37 \\
Voz24 & 0,46 & 0,24 & 2,08 & 1,06 \\
Voz25 & $-0,35$ & 0,43 & $-8,82$ & 10,49 \\
Voz26 & 0,18 & 0,25 & 7,81 & 10,43 \\
Voz27 & 1,42 & 0,40 & $-1,50$ & 0,30 \\
Voz28 & 0,67 & 0,23 & $-0,73$ & 0,32 \\
Voz29 & 0,88 & 0,30 & $-2,32$ & 0,67 \\
Voz30 & 0,43 & 0,23 & 1,68 & 0,89 \\
\hline & & & &
\end{tabular}


Tabela 6 - Parâmetros finais de Discriminação (a) e de Dificuldade (b) e seus Erros Padrões (EP) para o Módulo Voz.

\begin{tabular}{lcccc}
\hline Item & \multicolumn{2}{c}{ Discriminação } & \multicolumn{2}{c}{ Dificuldade } \\
\cline { 2 - 5 } & $\mathrm{a}$ & $\mathrm{EP}(\mathrm{a})$ & $\mathrm{b}$ & $\mathrm{EP}(\mathrm{b})$ \\
\hline Voz6 & 0,96 & 0,42 & $-3,14$ & 1,08 \\
Voz8 & 0,68 & 0,25 & $-1,15$ & 0,43 \\
Voz9 & 0,88 & 0,28 & $-0,76$ & 0,27 \\
Voz11 & 0,69 & 0,25 & 0,15 & 0,23 \\
Voz17 & 0,74 & 0,26 & $-1,30$ & 0,44 \\
Voz18 & 0,84 & 0,27 & $-0,85$ & 0,30 \\
Voz19 & 1,55 & 0,47 & $-1,52$ & 0,30 \\
Voz21 & 1,40 & 0,42 & $-1,51$ & 0,32 \\
Voz27 & 0,97 & 0,30 & $-0,55$ & 0,22 \\
Voz28 & 0,82 & 0,31 & $-2,46$ & 0,80 \\
\hline
\end{tabular}

Há apenas um item com parâmetro de dificuldade positivo. O item Voz19 foi o item com maior discriminação $(1,55)$, enquanto que o item Voz8 foi o item com a menor discriminação $(0,68)$. Já o item Voz11 foi o item com maior dificuldade $(0,15)$, enquanto que o item Voz6 foi o item com a menor dificuldade de acerto $(-3,14)$.

Figura 4 - Curvas Características dos Itens - CCl's (a) e Função de Informação dos Itens - Fll's (b)

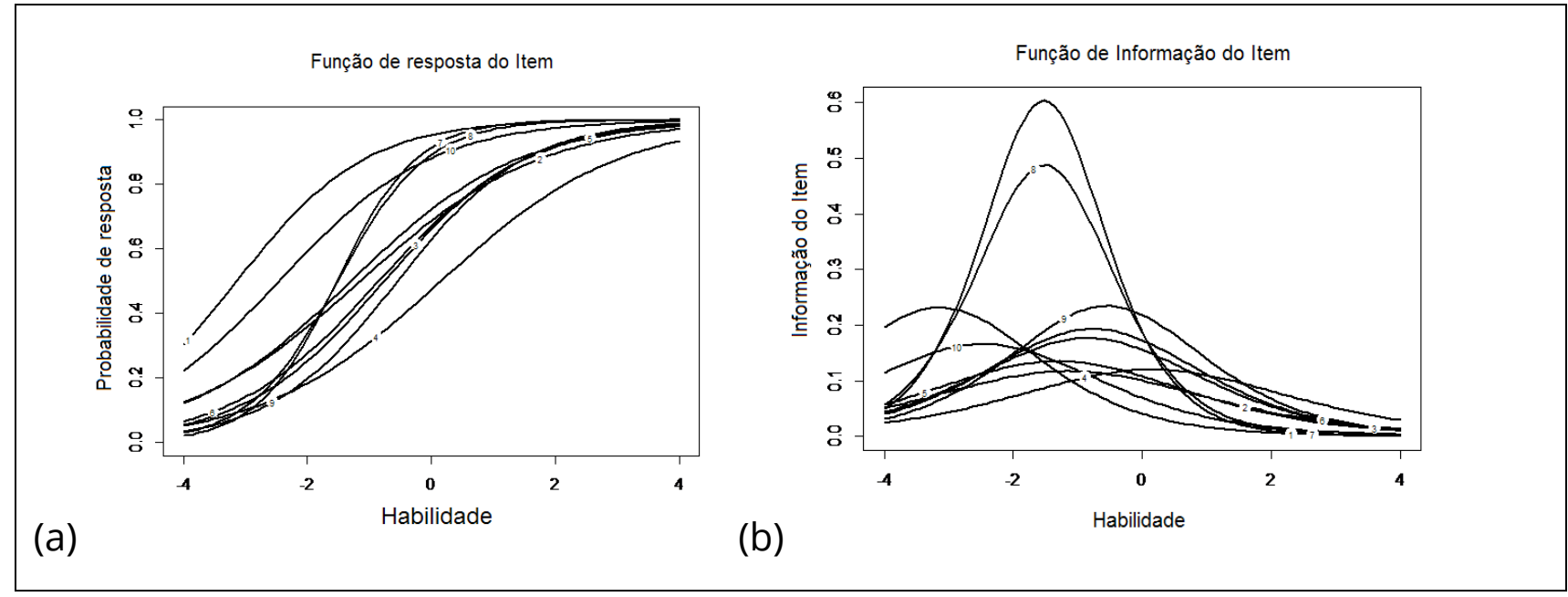

A FIT está apresentada na Figura 5, onde observa-se que a maior parte da informação concentra-se aproximadamente entre -4 e 0. Isso mostra que o questionário é mais adequado para medir indivíduos que têm baixa habilidade em identificar as expressões vocais. Isso ocorre justamente devido à falta de itens com parâmetro $b$ positivo, isto é, itens difíceis de serem acertados. Ou seja, faltam itens para avaliar os 
indivíduos que têm alta habilidade em identificar as emoções por meio de vozes. Isso também prejudica a estimação do nível de habilidade desses indivíduos, conforme verifica-se na Figura 6(b).

Figura 5 - Função de Informação do Teste

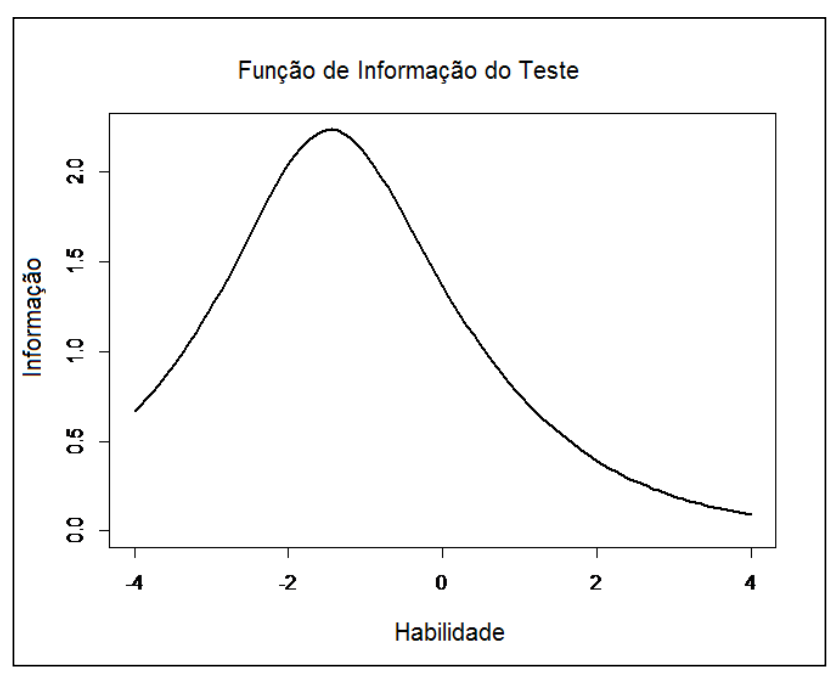

A Figura 6 apresenta o histograma (a) e o erro padrão (b) do traço latente. Observa-se que enquanto a maior parte da informação se concentra à esquerda, abaixo da média da escala (Figura 5), a maior parte dos indivíduos se concentra no meio, em torno da média da escala (Figura 6(a)).

Figura 6 - (a) Histograma e (b) Erro Padrão do traço latente

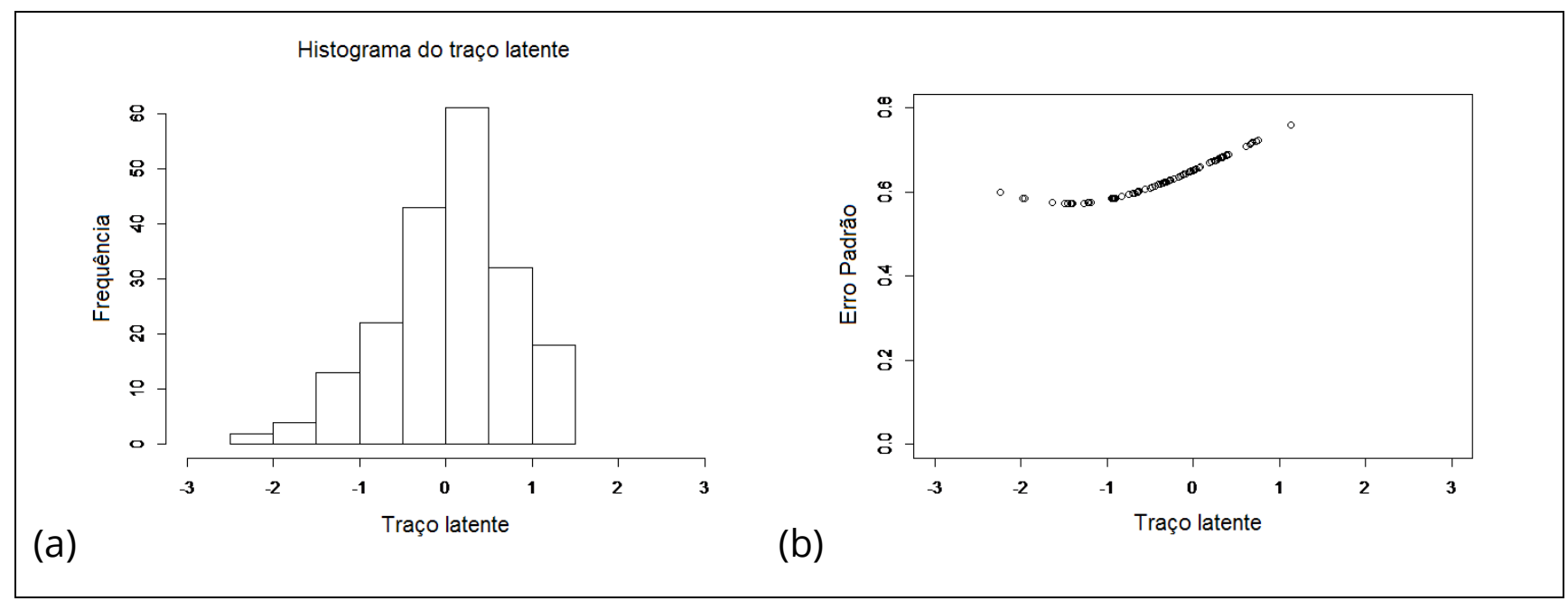




\section{CONCLUSÕES}

Esse trabalho apresentou uma avaliação de itens do ERI por meio do Modelo Logístico de Dois Parâmetros (ML2) da Teoria da Resposta ao Item. No decorrer da análise, vários itens tiveram que ser eliminados, tanto pela ausência da evidência de unidimensionalidade quanto pela falta de ajuste adequado segundo o ML2 da TRI. O construto Face apresentou um resultado um pouco melhor que o construto Voz, relacionado com a quantidade de itens fáceis com bom ajuste pelo ML2 da TRI.

No entanto, os resultados de ambos os construtos não foram satisfatórios para construir e validar uma escala por meio da TRI. Falta de itens de alta dificuldade, falta de precisão nas estimativas do traço latente e o elevado erro padrão do traço latente contribuíram para isso.

Quanto ao histograma e o erro padrão do traço latente (Figuras 3 e 6), foi observado um comportamento assimétrico em ambos os módulos. Isso ocorre devido à ausência de itens difíceis, os quais possibilitariam discriminação de indivíduos com traço latente acima do valor zero na escala.

Além disso, para o módulo Voz, o erro padrão (Figura 6b) é elevado em todo o contínuo da escala, não sendo menor que 0,55 em nenhuma parte da escala do traço latente. Isso revela que, embora o construto meça melhor o traço latente daqueles que têm menor habilidade em relação aos com maior habilidade, faltam itens de qualidade em todo o contínuo do traço latente, isto é, itens fáceis, médios e difíceis.

Os achados, segundo o modelo ML2 ajustado, sugerem que o ERI é uma ferramenta melhor empregada para avaliar indivíduos com habilidade baixa em reconhecimento de expressões faciais. O uso de TRI para estimativa de traço latente nesses indivíduos é um instrumento psicométrico importante.

A análise via TRI permitiu identificar itens com parâmetros inadequados para estimar o traço latente. Foram eliminados 12 itens do Módulo Face para que o pressuposto de unidimensionalidade fosse preenchido. O julgamento pelo especialista apontou a presença de unidades de ação mistas, correspondentes a mais de uma emoção. Os autores sugerem que o uso de expressões únicas, porém de baixa 
intensidade (expressões sutis), pode gerar itens de alta dificuldade e poder de discriminação.

Considerando trabalhar com os itens originalmente selecionados pelo ERI, as alternativas sugeridas são:

1. Fazer a validação, a construção e a interpretação da escala dos construtos Face e Voz por meio da Teoria da Resposta ao Item usando o ML3, modelo mais adequado quando o respondente tem a oportunidade de acertar a resposta mesmo sem saber, ou seja, o popular "chute". Para isso, sugere-se aumentar o tamanho da amostra e elaborando mais itens difíceis e que tenham característica unidimensional.

2. Aumentar consideravelmente o tamanho da amostra e verificar o ajuste por meio do Modelo de Resposta Nominal da TRI, que considera dados politômicos, ou seja, a resposta original do indivíduo. Embora o uso desse modelo não seja muito comum, pode-se abrir uma perspectiva para novas formas de avaliação do traço latente.

3. Verificar a possibilidade de ajuste por meio de Modelos Multidimensionais da TRI.

Os autores sugerem o uso da TRI como a ferramenta mais adequada para seleção de itens para compor futuros construtos voltados à avaliação da competência em percepção emocional (emotional perception competence). 


\section{REFERÊNCIAS}

ANDRADE DF, TAVARES HR, VALLE RC. Teoria da resposta ao item: conceitos e aplicações. São Paulo: ABE - Associação Brasileira de Estatística, 2000.

BAKER FB. Item response theory parameter estimation techniques, Marcel Dekker, New York, 1992.

BIEHL M, MATSUMOTO D, EKMAN P, HEARN V, HEIDER K, KUDOH T, TON V. Matsumoto and Ekman's Japanese and Caucasian Facial Expressions of Emotion (JACFEE): Reliability Data and Cross-National Differences. Journal of Nonverbal Behavior. 1997, Volume 21(1): 3.

DE AYALA RJ. The Theory and Practice of Item Response Theory. The Guilford Press, New York Wiley, 2009

EKMAN P, FRIESEN E. Facial Action Coding System: A Technique for the Measurement of Facial Movement. Consulting Psychologists Press, Palo Alto, 1978.

EKMAN P. Emotions revealed. New York: Times Book, 2003.

EKMAN P. A Linguagem das Emoções; São Paulo: Lua de Papel, 2011.

GAZZANIGA MS, IVRY RB, MANGUN GR. Neurociência cognitiva. Porto Alegre: Artmed. 2006.

KANG W, KIM G, KIM H, LEE SH. A influência da ansiedade no reconhecimento da emoção facial depende da categoria da emoção e da raça dos rostosalvo. Neurobiology experimental , 2019, 28(2): 261-269.

KNAPP ML, HALL JA. Nonverbal Communication in Human Interaction. 5th ed. Forth Worth, Tex.: Harcourt Brace Jovanovich, 2002

LORENZO-SEVA U, FERRANDO PJ. Manual of the Program Factor. Tarragona, Spain: Departament de Psicologia, Universitat Rovira i Virgili, 2013.

LUNDQVIST D, ÖHMAN A. Caught by the evil eye. In BARRETT LF, NIEDENTHAL PM, WINKIELMAN P. (Eds.), Emotion and consciousness 2005: 97-122.

MIGUEL FK. Psicologia das emoções: uma proposta integrativa para compreender a expressão emocional. Psico-USF, 2015, 20(1): 153-162.

MOREIRA JUNIOR FJ. A utilização da teoria da resposta ao item como ferramenta para a construção de escalas na área de gestão. Revista Gestão Organizacional, 2014, 6: 143-159. 
PICARD RW. Affective Computing. MIT Press, Cambridge, EUA, 1997.

PARTCHEV I. Package irtoys: Simple interface to the estimation and plotting of IRT models, 2013. R package version 0.1.7, 2015.

PLUTCHIK R. Emotions and life: Perspectives from psychology, biology and evolution. Washington, DC: American Psychological Association, 2002.

R DEVELOPMENT CORE TEAM. R: A Language and Environment for Statistical Computing. R Foundation for Statistical Computing, Vienna, Austria. ISBN 3-900051-070, 2013.

RIZOPOULOS D. Package Itm: Latent Trait Models under IRT, 2013.

PORTER S, TEN BRINKE L, WALLACE B. Secrets and Lies: Involuntary Leakage in Deceptive Facial Expressions as a Function of Emotional Intensity. Journal of Nonverbal Behavior, 2012, (36): 23-37.

SANTOS FA. Comunicação não verbal: identificação de emoções através de expressões faciais na prática da psicologia clínica. 2017. Universidade do Extremo Sul Catarinense, UNESC.

SCHERER KR. Component models of emotion can inform the quest for emotional competence. In G. Matthews, M. Zeidner, \& R. D. Roberts (Eds.), The science of emotional intelligence: Knowns and unknowns. New York, NY: Oxford University Press, 2007: 101-126.

SCHERER K, SCHERER U.Assessing the Ability to Recognize Facial and Vocal Expressions of Emotion: Construction and Validation of the Emotion Recognition Index. Journal of Nonverbal Behavior, 2011, (35): 305-326.

TIMMERMAN ME, LORENZO-SEVA U. Dimensionality assessment of ordered polytomous items with parallel analysis. Psychological Methods, 2011, 16(2): 20920.

VIEIRA, HAC. O padrão de trajetória visual para o reconhecimento de expressões faciais. 2015. Tese (Doutorado em Ciências do Comportamento) - Universidade de Brasília. Brasília. 